„Verfassungsschutz stellt Überwachung von Bürgerrechtler ein “, heise online v. 18.11.2008.

(http://www.heise.de/newsticker/meldung/Verfassungsschutz-stellt-ueberwachung-von-Buergerrechtler-ein-217246.html, zuletzt besucht am 27.10.2009).

17 In st. Rspr: BVerfGE 8,$326 ; 15,282 ; 25,365 ; 35,401 ; 37,150 ; 40,42$; 40, 274; 41, 323; 42, 130; 46, 178.

18 Briesach, Ein neuer Sicherheitsbegriff und dessen Herausforderungen an das BKA, in: Empirische Polizeiforschung X: Frevel/Asmus (Hrsg.), Einflüsse von Globalisierung und Europäisierung auf die Polizei, 2008, S. 31, Frankfurt a. Main.

19 v. Denkowski, Zur Einstufung sog. islamistischer Gefährder, Kriminalistik 5/2007, 325 (326).

$20 v$. Denkowski, Polizei-Nachrichtendienst-Trennungsgebot: Grenzen der Sicherheitsarchitektur, Kriminalistik 3/2008, 176 (182).

21 Middel, Innere Sicherheit und präventive Terrorismusbekämpfung, 2007, S. 69, Baden-Baden.

22 Paeffgen, Vernachrichtendienstlichung von Strafprozeß- (und Polizei-) recht im Jahr 2001, StV 4/2002, 336 (340).
23 Gustav Heinemann Initiative \& Humanistische Union, Das 2. Anti-TerrorPaket, in: Graubuch der Inneren Sicherheit, Dies. (Hrsg.), 2009, S. 24f., Berlin.

24 Feltes, Polizeiwissenschaft in Deutschland, Polizei \& Wissenschaft 4/2007, 2 (7) benutzt hierfür die Vokabel „Polizieren“.

25 Roggan/Kutscha, Handbuch zum Recht der Inneren Sicherheit, 2. Aufl., 2006, S. 177, Bonn.

26 Der Strafverteidigung erwachsen hieraus massive Problemstellungen, da (belastende) Personalbeweise oder Erkenntnisse von Nachrichtendiensten Sperrerklärungen unterliegen oder gar im Ausland vorliegen, so dass sie einer Überprüfung nicht zugänglich sind. Siehe dazu: Schünemann, Prolegomena zu einer jeden künftigen Verteidigung die in einem geheimdienstähnlichen Strafverfahren wird auftreten können, GA 2008, 314 (334).

27 Graulich, Justizgewährung und Geheimdienste, in: Terrorismus und Rechtsstaatlichkeit, Graulich/Simon (Hrsg.), 2007, S. 155, Berlin.

28 A.a.O.

29 Soine, Erkenntnisverwertung von Informanten und V-Personen der Nachrichtendienste im Strafverfahren, NStZ 3/07, 247 (252).

30 Roggan, Bürgerrechte \& Polizei 2/2004, 35 (36).

31 Ferse, OK - (K) eine Aufgabe für den Verfassungsschutz, KritV 2/1994, 256 (261); Roggan, Bürgerrechte \& Polizei 2/2004, 35 (39).

\title{
U-Haft Vollzug - Stiefkind der Strafrechtspflege
}

Prof.Dr. Heribert Ostendorf

In der wissenschaftlichen wie in der kriminalpolitischen Diskussion haben immer die Voraussetzungen für die Anordnung der U-Haft im Mittelpunkt gestanden. Der U-Haft-Vollzug hat wenig Interesse gefunden, er gilt als „Stiefkind der Strafrechtspflege“. Die gesetzlichen Voraussetzungen für die Anordnung der U-Haft bestimmen naturgemäß den U-Haft-Vollzug: werden die Möglichkeiten, die U-Haft anzuordnen sowie die Dauer zu verlängern, ausgeweitet, wächst die U-Haft-Population, umgekehrt verringert sich die U-Haft-Population, wenn die Voraussetzungen für die Anordnung und Verlängerung erschwert werden. Die Formulierung gesetzlicher Voraussetzungen der U-Haft, insbesondere auch die Einführung der richterlichen Entscheidungskompetenz ${ }^{1}$ sind das Ergebnis der Aufklärung und der Formulierung der Menschenrechte. So hat Cesare Beccaria in seinem Traktat über Verbrechen und Strafen (dei delitti e delle pene) im Jahre 1764 die Untersuchungshaft im Kapitel XXIX thematisiert. Er fordert, dass die Voraussetzungen für die U-Haft gesetzlich bestimmt sein müssen und beklagt, dass „man unterschiedslos Untersuchungshäftlinge und Strafgefangene in dasselbe Verlies wirft" (S. 134).

Während die rechtlichen Voraussetzungen für die Anordnung der U-Haft häufig vom Gesetzgeber verändert wurden, hat der Vollzug der U-Haft seit 1879 bis zum Jahre 2009 keine gesetzliche Änderung erfahren. Grundlage war durchgehend der $\$ 119$ StPO. Mit dem Strafvollzugsgesetz vom 16.3.1976 wurden zusätzlich das Arbeitsentgelt während der U-Haft ( $\$ 177)$ sowie der unmittelbare Zwang ( $\mathbb{1 7 8}$ i.V.m. den $\mathbb{\int}$ 94-101) gesetzlich geregelt. Ansonsten galt die Verwaltungsvorschrift der Untersuchungshaftvollzugsordnung vom 12.2.1953, in der Fassung vom 15.12.1976. Dieser Rechtszustand wurde seit Jahren als rechtsstaatliches Manko kritisiert. So hat die Strafvollzugskommission im Jahre 1971 gefordert, den Vollzug der Untersuchungshaft umfassend zu regeln. Allerdings hat das BVerfG in der Regelung des $\mathbb{} 119$ Abs. 3 StPO bislang (1981) „eine verfassungsrechtlich zureichende gesetzliche Grundlage für Einschrän- kungen grundrechtlicher Freiheiten des Untersuchungsgefangenen “ gesehen. $^{2}$

Einen ersten „Anlauf“ gab es bereits mit dem Amtlichen Entwurf eines Einführungsgesetzes zum Entwurf eines Allgemeinen Deutschen Strafgesetzbuches des Reichsministeriums aus dem Jahr 1929. Insbesondere infolge der Entscheidung des BVerfG zur Verfassungswidrigkeit der gesetzlichen Nichtregelung des Strafvollzugs (BVerfGE $33,1)$ wurden Gesetzesentwürfe aus der Wissenschaft und Praxis und schließlich auch aus dem Bundesjustizministerium eingebracht: 1981 der Entwurf des Strafrechtsprofessors Jürgen Baumann, 1982 der Entwurf der Anstaltsleiter, 1985 der Entwurf der Arbeitsgemeinschaft sozialdemokratischer Juristen (AsJ). In diesem zeitlichen Kontext steht auch die Habilitationsschrift von Manfred Seebode „Der Vollzug der Untersuchungshaft“ aus dem Jahr 1985. 1986 legte das Bundesjustizministerium einen Arbeitsentwurf vor, 1996 einen vorläufigen Referentenentwurf. Ein Gesetzentwurf zur Regelung des Vollzugs der Untersuchungshaft folgte im Jahr 1999³, dessen Verabschiedung insbesondere auch aufgrund fiskalischer Einwände der Länder hinausgezögert wurde - bis zum Inkrafttreten der sog. Förderalismusreform und der Zuständigkeitsverlagerung auf die Bundesländer. Die bisherigen Gesetzesentwürfe werden zusammenfassend von Friedrich in seiner Dissertation „Die Normierung des Untersuchungshaftvollzugs" mit der Ausarbeitung eines Alternativentwurfs (2004) diskutiert und bewertet.

Prof. Dr. Heribert Ostendorf, Forschungsstelle für Jugendstrafrecht und Kriminalprävention an der Christian-Albrechts-Universität Kiel

\section{Fußnoten:}

1 S. hierzu Ollinger, Die Entwicklung des Richtervorbehalts im Verhaftungsrecht, 1997; hierauf stützend Amelung Jura 2005, 447 ff.

2 BVerfGE 57, 170 (177); s. auch bereits BVerfGE 35, 311 (316).

3 BR-Drucks. $249 / 99$. 